\title{
Toward Understanding the Effects of Cognitive Styles on Collaboration in Multiplayer Games
}

Sultan A. Alharthi Play \& Interactive Experiences for Learning Lab New Mexico State University Las Cruces, NM, USA

salharth@nmsu.edu

\section{George E. Raptis}

Human Opsis

Patras, Greece

raptisg@upnet.gr

Christina Katsini

Human Opsis

Patras, Greece

katsinic@upnet.gr
Igor Dolgov

Department of Psychology

New Mexico State University

Las Cruces, NM, USA

id@nmsu.edu

\section{Lennart E. Nacke}

$\mathrm{HCl}$ Games Group, Games

Institute, University of Waterloo

Waterloo, ON, Canada

lennart.nacke@acm.org

Zachary O. Toups

Play \& Interactive Experiences

for Learning Lab

New Mexico State University

Las Cruces, NM, USA

z@cs.nmsu.edu

Permission to make digital or hard copies of part or all of this work for personal or classroom use is granted without fee provided that copies are not made or distributed for profit or commercial advantage and that copies bear this notice and the full citation on the first page. Copyrights for third-party components of this work must be honored. For all other uses, contact the owner/author(s).

Copyright held by the owner/author(s).

CSCW'18 Companion, November 3-7, 2018, Jersey City, NJ, USA ACM 978-1-4503-6018-0/18/11.

https://doi.org/10.1145/3272973.3274047

\begin{abstract}
In multiplayer games, players need to coordinate action to succeed. This paper investigates the effect of cognitive styles on performance of dyads engaged in collaborative gaming activities. 24 individuals took part in a mixed methods user-study; they were classified as field dependent (FD) or independent (FI) based on a cognitive style elicitation instrument. Three groups of teams were formed, based on the cognitive style of each team member: FD-FD, FD-FI, $\mathrm{Fl}-\mathrm{Fl}$. We examined performance in terms of game completion time, cognitive load, and player experience. The analysis revealed that FD-FI cognitive style had an effect on the performance and the mental load. We expect the findings to provide useful insight for practitioners and researchers on improving team collaboration in different contexts, such as learning, eSports, and disaster response.
\end{abstract}

\section{Author Keywords}

Cognitive styles, teams, collaboration, planning, team formation, multiplayer games, user study.

\section{Introduction}

Effective teamwork is characterized by having a shared understanding of objectives and collaboration skills. In distributed multiplayer games, players need to constantly coordinate actions with each other to succeed as a team, hence, there is a need to understand collaboration in games 


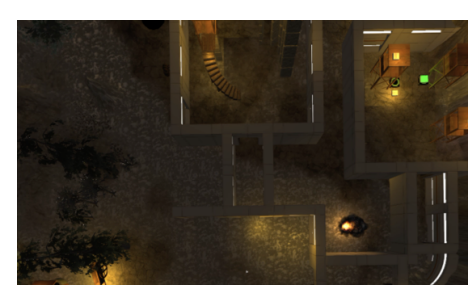

Figure 1: The top-down map of the game allows players to see part of the play space to develop strategies and plans.

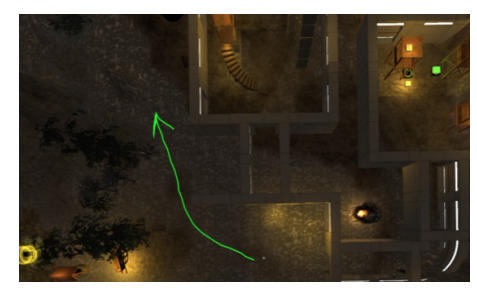

Figure 2: Players use the mouse cursor to annotate on the map to establish plans.

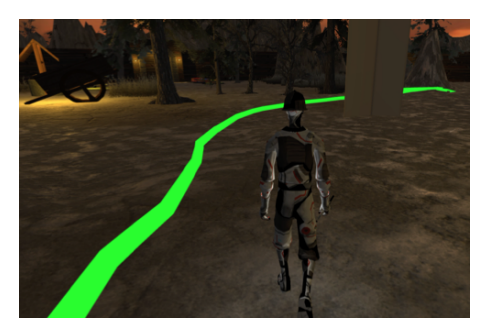

Figure 3: Annotations are made visible directly on the gameworld, enabling its use during play. and how it can be improved $[2,10]$. To accomplish game objectives, players typically process information provided by the game and their co-players [14]. Considering that individuals follow different approaches to process information [6], cognitive styles are expected to play an important role in the success of teamwork and team formation in games.

While prior studies have investigated the effect of cognitive styles in single-player games [7, 8], no research has examined cognitive styles in collaborative games. We explore how cognitive styles impact dyadic collaboration in games. The paper presents our game, the Team Coordination \& Planning Game ( $\mathrm{TeCP}$ ) and an empirical evaluation of how cognitive styles affect team performance, cognitive workload, and experience. We expect our findings to provide useful insights for practitioners, researchers, and implications for the broader CSCW community on improving team collaboration and formation in different contexts such as learning, eSports, and disaster response.

\section{Background}

In cognitive psychology, cognitive styles describe how people acquire, perceive, and process information [6]. One established and validated cognitive style is Field Dependence / Independence (FD-I) [13]. According to FD-I, people are characterized either as field-dependent (FD) or fieldindependent $(\mathrm{FI})$, based on their ability to extract visual information in complex scenes [13]. A person characterized as FD tends to prefer personal orientation, be holistic, have difficulties in distinguishing details from other information and perform better on inductive tasks; a person classified as FI tends to prefer impersonal orientation, be analytical, pay attention to details and easily separate simple elements and structures from surrounding context.
In teamwork, performance can be maximized when teams organize their activities, synchronize their effort, and maintain shared mental models and situation awareness [3, 4, 14]. During collaborative activity, teams plan action by gathering relevant information, analyzing it to establish strategy, and make sense of the situation, which leads to accomplishing a shared goal $[2,14]$. Such work involves identifying, searching, filtering, sharing, and synthesizing information, hence, individual differences in cognitive style have the potential to impact the success of collaboration and planning in teams $[7,8,13]$.

Collaboration in games is enabled through different communication channels, such as voice, text, and cooperative communication mechanics-game mechanics that support communication and enable shared references in gameworlds [10]. While teamwork and collaboration in games have been researched previously (e.g., [2, 11]), no research has yet examined the role of cognitive styles.

\section{Method and Participants}

To identify the effects of FD-I cognitive style on team collaboration in games, we selected $T e C P$, a two-person cooperative game in which players' avatars are physically separated in the gameworld [1, 2, 9]. The main objective in the game is for players to complete a set of collaborative tasks (e.g., opening doors for each other, finding and placing cubes in their associated places) to successfully escape a maze (Figure 1). Players in $\mathrm{TeCP}$ are able to move their avatars in all directions and carry, place, and stack cubes to complete the different collaborative tasks. Dependences in the game force players to work together by communicating and collaborating. Players communicate and plan using a combination of text and voice chat with an annotation cooperative communication mechanic - freely drawn visual lines and shapes on top of the gameworld (Figures $2 \& 3$ ) [2]. 


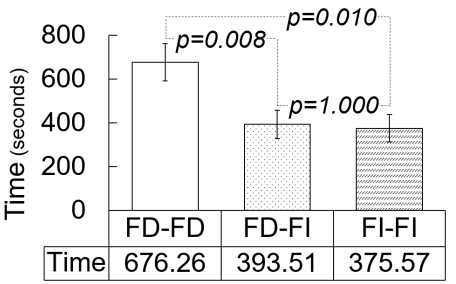

Figure 4: FD-FD group needed significantly more time than FD-FI and $\mathrm{FI}-\mathrm{FI}$ groups to complete the game.

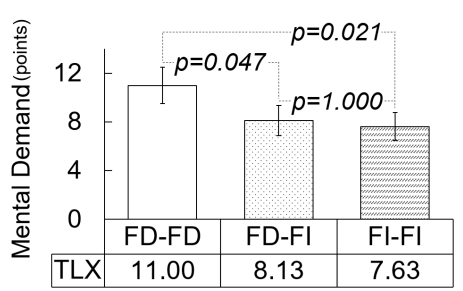

Figure 5: FD-FD group found the task significantly more mental demanding than the other groups.

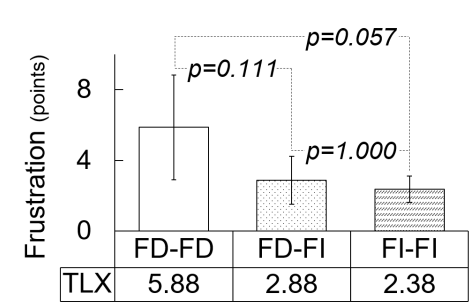

Figure 6: FD-FD group found the task marginally more frustrating than the FI-FI group.
Hypotheses and Procedure

Based on our motivation and study of related work, we formed and tested the following hypotheses, for which we were suspecting that the main effects would reveal which team type (FD-FD, FD-FI, FI-FI) performs best:

(H1): there is significant difference in the game completion time between the different team types;

(H2): there is significant difference in the cognitive load between the different team types; and

(H3): there is significant difference in the player experience between the different team types.

We recruited 24 individuals ( 4 female) with an average age 22.4 years $(s d=2.9)$, who were all experienced videogame players (play more than 1 hour every day). All participants were provided with an overview of the study and then provided consent; we followed a two-step study approach. First, participants completed a demographics questionnaire (5 minutes) and undertook Group Embedded Figures Test (15 minutes) [13] to classify them either as FD or FI. Next, we formed three balanced groups of all combinations of cognitive style (i.e., FD-FD, FD-FI, FI-FI); each group had four dyads. As a second step, each team was invited to SpiderNet Internet Café in Patras, Greece to play the game (5 minutes practice followed by a 15 minutes play session) at a mutually agreed day and time. Each participant was part of only one session in this between-subject study. After the play session ended, the participants completed the NASA

TLX to assess workload [5] (5 minutes), which provides an overall score for how demanding the task was, along with subgroup scores; and the PXI to assess player experience (5 minutes) [12].

\section{Preliminary Results}

For each hypothesis, we ran a one-way ANOVA test, which met all the required assumptions. Workload and player experience, both subjective metrics, were evaluated at the team level, by averaging the scores of the team members. The analysis revealed:

(H1): FD-FD group needed significantly more time than FD-FI and FI-FI to complete the game (Figure 4);

(H2): FD-FD group assessed the task as significantly more mentally demanding on the TLX subgroup than FD-FI and FI-FI (Figure 5) and marginally more frustrating than FI-FI (Figure 6); however, no significant differences were found for overall workload.

(H3): no significant differences were found.

As an additional data point, we observed that all teams used verbal communications while annotating critical information (e.g., path, target position) on the map.

\section{Discussion}

People characterized as FD typically face difficulties in distinguishing details within complex scenes, which appears to hold for $T e C P$. As a result, the FD-FD groups had poor performance and increased mental workload, despite communicating verbally and with annotations. The groups that had at least one player characterized as FI performed better, which we attribute to the ability of such players to identify game targets and find a way to accomplish the goals within the visually complex background. In multiplayer collaborative games that require demanding visual tasks, cognitive styles should be considered as a human factor that influences performance. In our example, grouping players characterized as FD and FI will have an increased performance in time-dependent tasks and demanding/stressful contexts, such as eSports and disaster response.

\section{Conclusion and Future Work}

We presented the preliminary results of a small-scale userstudy on the effects of cognitive styles on collaboration in 


\section{Acknowledgments}

We thank SpiderNet Internet Café in Patras, Greece for providing their space and equipment, contributing to the ecological validity of the study.

Lennart Nacke thanks NSERC Discovery (RGPIN-2018-06576, Developing and Analyzing Immersive Game Technologies and Components for Motivation, Wellbeing, and Guidance) NSERC CREATE SWaGUR, SSHRC (895-2011-1014, IMMERSe), CFI (35819, JELF) for funding his research team.

This material is based upon work supported by the National Science Foundation under Grant Nos. IIS-1651532 and IIS-1619273. distributed multiplayer games. The results indicate that cognitive styles should be considered as a human factor when forming dyads in collaborative games, which are based on time-dependent and visually complex tasks, to increase team performance. Future work includes a) increasing sample size, b) further investigating the effects of cognitive styles on other game parameters, c) determining if game-specific training can influence ability to distinguish elements in complex scenes, and d) considering other cognitive styles and games.

\section{REFERENCES}

1. Sultan A. Alharthi, Ruth C. Torres, Ahmed S. Khalaf, and Zachary O. Toups. 2017. The Maze: Enabling Collaborative Planning in Games Through Annotation Interfaces. In Ext. Abs. An. Symp. Comp.-Human Interaction in Play (CHI PLAY '17). ACM, 615-620.

2. Sultan A. Alharthi, Ruth C. Torres, Ahmed S. Khalaf, Zachary O. Toups, Igor Dolgov, and Lennart E. Nacke. 2018. Investigating the Impact of Annotation Interfaces on Player Performance in Distributed Multiplayer Games. In Proc. SIGCHI Conf. Human Factors in Comp. Sys. (CHI '18). ACM, 314:1-314:13.

3. Mica R. Endsley. 1995. Toward a theory of situation awareness in dynamic systems. Human Factors 37, 1 (1995)

4. Dedre Gentner and Albert L. Stevens. 1983. Mental Models. Lawrence Earlbaum Associates, Hillsdale, NJ, USA.

5. S. G. Hart and L. E. Staveland. 1988. Development of NASA-TLX (Task Load Index): Results of empirical and theoretical research. In Human Mental Workload, P. A. Hancock and N. Meshkati (Eds.). 239-250.

6. Maria Kozhevnikov. 2007. Cognitive styles in the context of modern psychology: Toward an integrated framework of cognitive style. Psychological Bulletin 133, 3 (2007), 464

7. George E. Raptis, Christos Fidas, and Nikolaos Avouris. 2017. Cultural Heritage Gaming: Effects of Human Cognitive Styles on Players' Performance and Visual Behavior. In
Adjunct Pub. 25th Conf. User Modeling, Adaptation and Personalization (UMAP '17). ACM, 343-346.

8. George E. Raptis, Christos A. Fidas, and Nikolaos M. Avouris. 2016. Do Field Dependence-Independence Differences of Game Players Affect Performance and Behaviour in Cultural Heritage Games?. In Proc. 2016 An. Symp. Comp.-Human Interaction in Play (CHI PLAY '16). ACM, 38-43.

9. Zachary O. Toups, William A. Hamilton, and Sultan A. Alharthi. 2016. Playing at Planning: Game Design Patterns from Disaster Response Practice. In Proc. 2016 An. Symp. Comp.-Human Interaction in Play (CHI PLAY' 16). ACM, 362-375.

10. Zachary O. Toups, Jessica Hammer, William A. Hamilton, Ahmad Jarrah, William Graves, and Oliver Garretson. 2014. A Framework for Cooperative Communication Game Mechanics from Grounded Theory. In Proc. 1st An. Symp. Comp.-human Interaction in Play (CHI PLAY' 14). ACM, 257-266.

11. Deepika Vaddi, Zachary O. Toups, Igor Dolgov, Rina Wehbe, and Lennart E. Nacke. 2016. Investigating the Impact of Cooperative Communication Mechanics on Player Performance in Portal 2. In Proc. 42nd Graphics Interface Conf. (GI '16). 41-48.

12. Vero Vanden Abeele, Lennart E. Nacke, Elisa D. Mekler, and Daniel Johnson. 2016. Design and Preliminary Validation of The Player Experience Inventory. In Proc. 2016 An. Symp. Comp.-Human Interaction in Play Comp. Ext. Abs. (CHI PLAY 16). ACM, 335-341.

13. H. A. Witkin, C. A. Moore, D. R. Goodenough, and P. W. Cox. 1977. Field-Dependent and Field-Independent Cognitive Styles and Their Educational Implications. Review of Educational Research 47, 1 (1977), 1-64.

14. Jason Wuertz, Sultan A. Alharthi, William A. Hamilton, Scott Bateman, Carl Gutwin, Anthony Tang, Zachary O. Toups, and Jessica Hammer. 2018. A Design Framework for Awareness Cues in Distributed Multiplayer Games. In Proc. SIGCHI Conf Human Factors in Comp. Sys. (CHI '18). ACM, 243:1-243:14. 